\title{
Reduction of Stresses and Mass of an Engine Rubber Mount Subject to Mechanical Vibrations
}

\author{
Omar Dávalos ${ }^{1}$ - Uzziel Caldiño-Herrera ${ }^{1}$ - Delfino Cornejo-Monroy ${ }^{1}-$ \\ Oscar Tenango-Pirin 1 - Juan Carlos García2,* - M.A. Basurto-Pensado² \\ 1Universidad Autónoma de Ciudad Juárez, México \\ ${ }^{2}$ Centro de Investigación en Ingeniería y Ciencias Aplicadas, Universidad Autónoma del Estado de Morelos, México
}

\begin{abstract}
A rubber engine mount (EM) is a mechanical coupling between the engine and the chassis, and its main function is to diminish, in the chassis, the amplitude of vibrations caused for the engine operation. Such vibrations cause discomfort for vehicle passengers and reduce the EM lifetime. To increase the comfort of vehicle passengers and the lifetime of the EM, this paper presents an EM optimization by means of reducing three main criteria: the EM mass, the displacements transmitted to the chassis, and the mechanical stress in the EM rubber core. For carrying out the EM optimization, the optimum global determination by linking and interchanging kindred evaluators (GODLIKE), assisted by artificial neural networks (ANN) and finite element method (FEM), was used. Because of the optimization process, a reduction greater than $10 \%$ was achieved in the three criteria in comparison with a baseline design. The frequency responses were compared and showed that although the optimization was carried out for the range of $5 \mathrm{~Hz}$ to $30 \mathrm{~Hz}$ the trend of reduced responses continues beyond this range. These results increased the comfort of vehicle passengers and the lifetime of the EM; in addition, the reduction of mass diminishes its production costs.
\end{abstract}

Keywords: multi-objective optimization, vehicle engine mount, ANN, FEM, global optimum determination

\section{Highlights}

- An engine mount (EM) was optimized using the integration of optimization algorithms, ANN, and FEM.

- For the EM optimization, the global optimum determination by linking and interchanging kindred algorithm was used.

- The optimization is focused on the reduction of EM mass, displacements, and stress responses under mechanical vibration.

- The engine operation frequencies were obtained via experimental measurements.

- The errors of ANN model predictions were less than $5 \%$.

- The FEM model was validated by the experimental measurements of natural frequencies.

\section{INTRODUCTION}

A current trend in the automotive industry is the tendency to downsize the components to increase vehicle power capacity, to simplify the manufacturing process and reduce production costs, among other features. This downsizing is only possible if the modifications do not compromise passenger comfort or affect the performance of the automotive systems. Thus, there is a simultaneous need to find automotive components susceptible to improvements and strategies capable of optimizing new designs. One of these automotive parts is the EM, whose main function includes the attenuation of engine vibrations, by means of the reduction of stiffness, and to support the weight of the engine [1] and [2].

The EM consists of a cylindrical steel structure fixed to a rubber core, and it is exposed to forced vibrations caused by the engine operation [3] to [5]. The forced vibrations could cause failure due to fatigue of the EM rubber core, limiting the EM lifetime to five or six years at most. It should be noted that the damage caused by the cyclic load on the steel section of the EM parts is minimal [6] and [7]. Under these conditions, a good design of an EM mainly involves the reduction of the displacements transmitted from engine to chassis, the increasing of the lifetime through the reduction of mechanical stresses and the reduction of weight. This is a complicated task due to the interaction of the design variables. Heuristic optimization techniques are a good option when solving this type of engineering problems.

Researchers in different engineering fields have analysed these types of problems using optimization techniques. Pérez-Carabaza et al. [8] optimized the trajectory of an unmanned aerial vehicle using search algorithms of minimum time. Daróczy et al [9] optimized the aerofoil geometry of an H-rotor employing computational fluid dynamics and genetic algorithms; they increased the H-rotor power coefficient from 0.40 to 0.48 . Cheng et al. [10] improved a cuckoo search algorithm applied to vibration fault diagnosis. In another study [11], a soft optimization based on differential evolution was applied to attenuate the vane-rotor shock interaction in high-pressure turbines. They achieved attenuation above $60 \%$ without stage-efficiency abatement. Rai and Barman [12] applied simulated annealing and real 
coded genetic algorithms to optimize the design of a spur gear. They obtained reductions of $14.1 \%$ and $16.6 \%$ of material by using the simulated annealing algorithm and the real coded genetic algorithm, respectively. In [13], the non-dominated sorting genetic algorithm was used to improve the efficiency, and the output power of a piston compressed air engine. They obtained, as an optimized result, an efficiency of $31.17 \%$ when the output power was $2 \mathrm{~kW}$. However, for the case of multi-objective optimization problems, GODLIKE has been used to find one common optimal solution [14].

Regarding EM optimization, several works have employed heuristic techniques. In Ahn et al. [15] an optimization of an EM, by means of an enhanced genetic algorithm with a simplex method and sequential quadratic programming, was employed. The aim of the study was to reduce both the notch depth and the resonance peaks. Both algorithms converged in the presence of selected constraints for the design parameters which improved the forces of transmission to the vehicle by about $30 \%$ due to reduction of notch depth and resonance peaks. Lee and Kim [16] used a micro-genetic algorithm, ANN, and FEM to reduce the mechanical stresses and to increase the life cycles of an elastomeric EM. They obtained a $24 \%$ reduction of stresses and an increment greater than $100 \%$ in the fatigue life cycles. Furthermore, they compared their results against a simple genetic algorithm (GA), showing that a micro-GA performs better than a simple GA. In Zhao et al. [17], a topological optimization was performed to reduce the weight of an EM, maximizing the natural frequencies and increasing the life cycles. They obtained $1.5 \times 10^{6}$ cycles, more than three times the initial target. Alvarado-Iniesta et al. [18] used memetic genetic programming to optimize an engine mount under static load conditions, using a surrogate method and FEM. They reported a reduction of EM weight and stresses.

In this work, a multi-objective design optimization of an EM is proposed using GODLIKE assisted by ANN and FEM. The optimization aim was to reduce the mass of the EM and to reduce the displacements and stresses under mechanical vibrations. Three objective functions were defined, including a target and a penalty factor for each objective function. Experimental measurements were accomplished to identify the mechanical vibration frequency range of the engine; later, this range was used as a boundary condition in commercial FEM software to perform frequency response simulations. ANN was used as a surrogate method within GODLIKE to predict the rubber core mass, displacement, and stress responses. The ANN was trained with a database generated from a central composite design of the experiments. Measurement of the EM natural frequency was used to validate the numerical model, and this was used to compute the EM frequency response using FEM. This multiobjective optimization is a novel way to link three different algorithms (GODLIKE, ANN, and FEM) to improve its performance and reach a fast optimization methodology of EM, considering stress and displacement responses under mechanical vibrations. Using another way for the optimization process, the calculation of these responses requires a large amount of time for the numerical computations. However, in this multi-objective optimization algorithm, to reduce the computation time, a surrogated method, like ANN, is employed. Thus, the cases of FEM computations are reduced according to the size of the database used in ANN training. Furthermore, the ANN database was constructed using a design of experiments which is helpful in attaining convergence, further reducing the time of optimization. The proposed optimization methodology is applied to a specific EM model, however; the methodology can be applied to other EM types considering its characteristics.

\section{METHODS}

\subsection{Optimization Methodology}

This work is focused on the numerical optimization of an EM, but the manufacturing of the optimized EM is out of the scope. The EM used in this investigation is located on the front right side of a 4-cylinder, 2.4 L Toyota internal combustion engine. One of its sides is attached to the chassis, and the other side supports the engine. The materials of which it is made are steel and rubber. The steel portion of the EM includes a cylindrical cover, a ring between the cylindrical cover and the rubber core, and a metal bushing between the rubber core and the bolt that fixes the engine. The rubber core has two lobe-shaped holes, one lateral tip at each side and a conical-shape around the metal bushing. The typical failure of the EM occurs in the rubber core. The crack propagation path extends along the neck of the lobe and around the conical shape, as shown in Fig. 1. Generally, the damage in the EM steel parts is negligible.

The sequence of the optimization methodology is shown in Fig. 2. First, the frequencies of the forced vibration caused by the engine operation are gotten through experimental measurements. These measured frequencies will show the range of the EM 


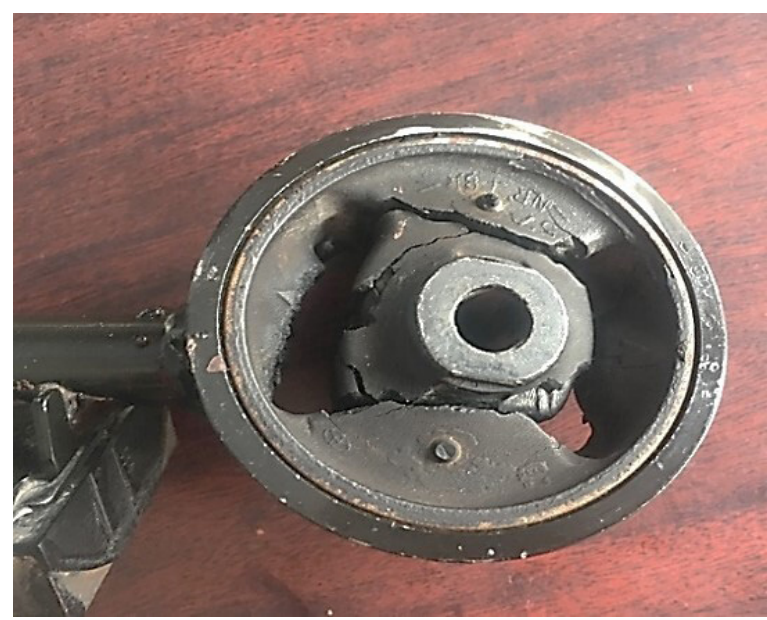

Fig. 1. Failed of the rubber core in an EM

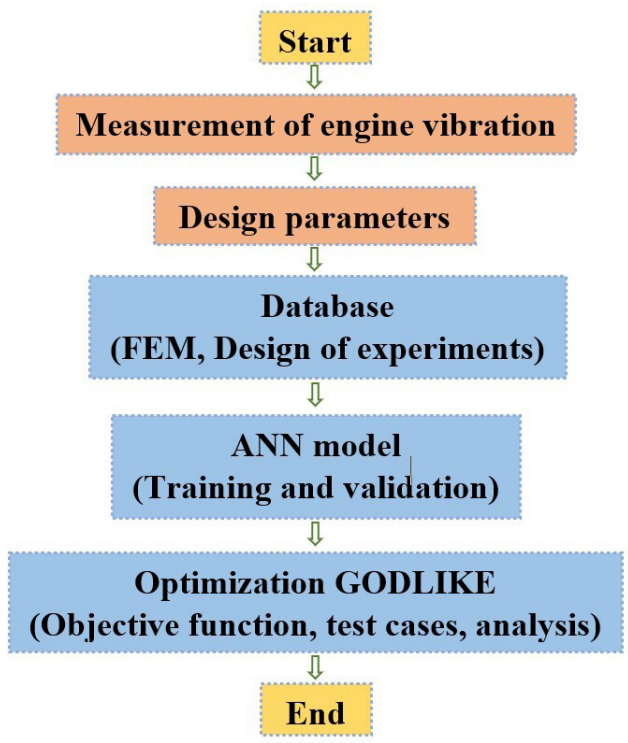

Fig. 2. Optimization methodology

excitation frequencies in which responses will be computed. Then, the design parameters are defined based on the EM geometry and the optimization requirements, which are the reduction of three criteria: EM mass, displacement response, and stress response. Afterwards, a database is generated using a central composite design of experiments and the values of stress and displacement to complete the database are computed using frequency response analysis trough FEM simulations. Once the database was generated, it was used for the training and the validation of an ANN model, which could accurately predict the rubber core mass and both displacement and stress responses. The final part is the application of the GODLIKE algorithm in the optimization of the design of the EM. The detailed description of each part of the optimization methodology is presented in the next sections.

\subsection{Measurement of Engine Mechanical Vibrations}

A triaxial G-Link-200 Microstrain ${ }^{\circledR}$ wireless accelerometer was placed over the engine block (Fig. 3 ) to measure the acceleration amplitude of engine mechanical vibrations. The acquired signal from the accelerometer was transmitted to a WSDA ${ }^{\circledR}-200$ USB Microstrain ${ }^{\circledR}$ receiver connected to a personal computer. The acceleration measurements were carried out at $1500 \mathrm{rpm}, 2000 \mathrm{rpm}, 2500 \mathrm{rpm}, 3000$ rpm, and ralenti conditions (around $800 \mathrm{rpm}$ to 900 rpm).

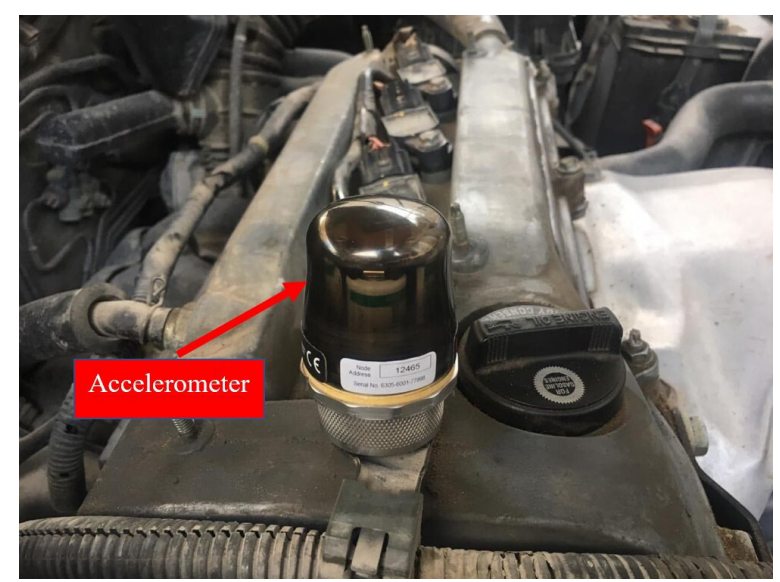

Fig. 3. Mounting of the accelerometer over the engine block

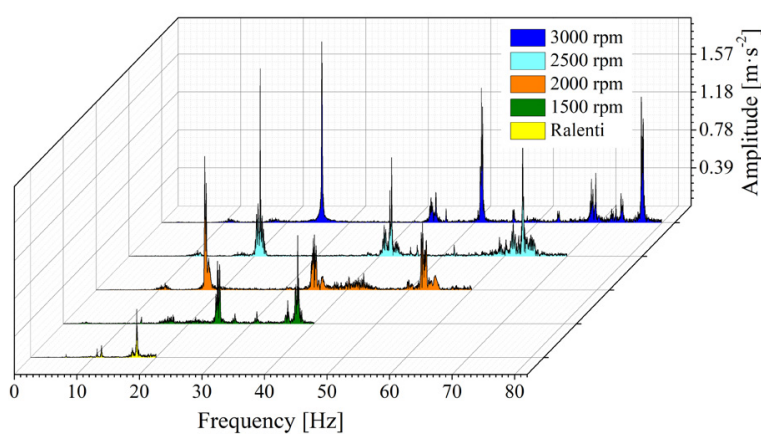

Fig. 4. Engine mechanical vibration spectrum at ralenti, 1500 rpm, 2000 rpm, 2500 rpm, and 3000 rpm

The acceleration signal of engine mechanical vibration in the time domain was converted to the frequency domain using the fast Fourier transform. The results of measured frequencies are presented in Fig. 4. At ralenti, the measured engine frequency was $5.7 \mathrm{~Hz}$ and increased with increasing engine revolutions. The maximum frequency was $25.6 \mathrm{~Hz}$ 
at $3000 \mathrm{rpm}$. Based on these findings, the frequency range for the FEM computations and optimization was set from $5 \mathrm{~Hz}$ to $30 \mathrm{~Hz}$.

\subsection{Parameterization}

For the EM optimization, only the part that holds the engine was considered since it is where failure occurs within the rubber core element. The metal parts were considered in the optimization process to reduce the total mass of the EM.

A total of eight geometrical variables were selected as design parameters: the outer diameter of the metal ring $\left(V_{1}\right)$, the external diameter of the rubber core $\left(V_{2}\right)$, the internal diameter of the rubber core $\left(V_{3}\right)$, the external diameter of the cylindrical cover $\left(V_{4}\right)$, the separation between the internal diameter of the rubber core and the lateral tip $\left(V_{5}\right)$, the internal diameter of the thickness of rubber wall $\left(V_{6}\right)$, the separation between tips of rubber lobes $\left(V_{7}\right)$ and the base diameter of a rubber cone $\left(V_{8}\right)$ around the metal bushing. Fig. 5 illustrates the location of the design parameters.

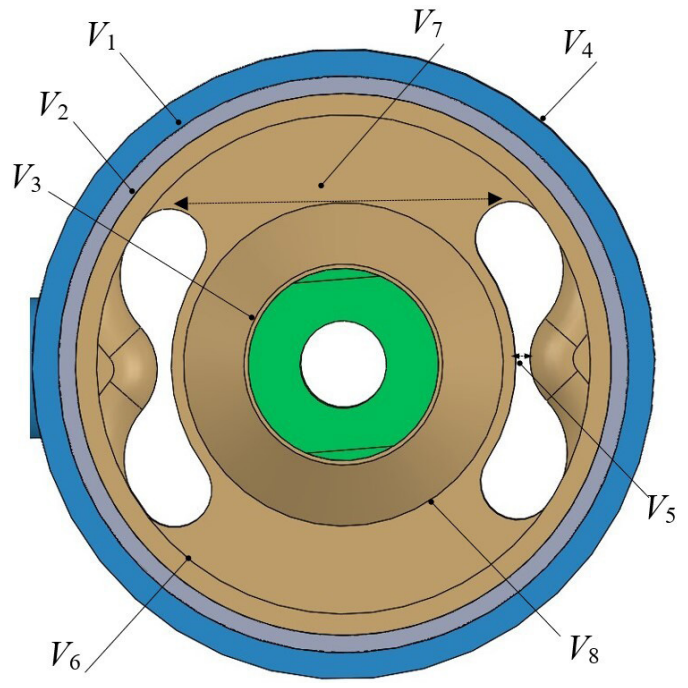

Fig. 5. EM design parameters:

$$
V_{1}, V_{2}, V_{3}, V_{4}, V_{5}, V_{6}, V_{7} \text {, and } V_{8}
$$

The lower and upper limits of the design variables are presented in Table 1. These limits were defined to avoid EM geometry interferences and available space to install EM into the engine bay.

\subsection{Database and Frequency Response Analyses}

To complete the database for ANN training and validation, the EM geometrical values of Table 1 were used to compute the frequency harmonic response using FEM by means of Ansys software. In this way, the stress, based on the von Mises stress theory, and displacements responses caused by engine mechanical vibrations were computed. Firstly, a load, considered as sinusoidal, with a frequency range between $5 \mathrm{~Hz}$ and $30 \mathrm{~Hz}$ with a step of $1 \mathrm{~Hz}$ was used. To compute the EM response to higher harmonics excitation, the frequency range was extended until $100 \mathrm{~Hz}$.

Table 1. Design variables bounds

\begin{tabular}{lcc}
\hline Variable & Lower limit [mm] & Upper limit [mm] \\
\hline$V_{1}$ & 64 & 68 \\
\hline$V_{2}$ & 60 & 64 \\
\hline$V_{3}$ & 20 & 24 \\
\hline$V_{4}$ & 72 & 76 \\
\hline$V_{5}$ & 1 & 3 \\
\hline$V_{6}$ & 57 & 61 \\
\hline$V_{7}$ & 36 & 40 \\
\hline$V_{8}$ & 34 & 40 \\
\hline
\end{tabular}

The frequency response analysis is based on the equation of motion for a mechanical vibration which is written as [19]:

$$
[\mathbf{M}]\{\ddot{\mathbf{u}}\}+[\mathbf{C}]\{\dot{\mathbf{u}}\}+[\mathbf{K}]\{\mathbf{u}\}=\{\mathbf{f}(t)\},
$$

where $\mathbf{M}$ is the mass matrix, $\mathbf{u}$ is the nodal displacement vector, $\mathbf{C}$ is the damping matrix, $\mathbf{K}$ is the stiffness matrix, and $\mathbf{f}(t)$ is the forcing vector or the dynamic load applied to EM. This dynamic load is a harmonic function:

$$
\mathbf{f}(t)=\mathbf{F} \sin (\omega t),
$$

where $\boldsymbol{\omega}$ is the frequency and $\mathbf{F}$ is the force vector.

The FEM computations assumed the rubber material as linear elastic to simplify the frequency response study. However, this assumption is limited to small deformations. For this research, to guarantee the validity of the FEM computations, experimental validation with the EM natural frequencies was implemented.

The property of materials, rubber, and metal, are described in Table 2.

The baseline EM model was discretized using tetrahedral elements with sizes between $1.2 \mathrm{~mm}$ and $1.8 \mathrm{~mm}$, resulting in 401,613 finite elements (Fig. 6). For each node, six degrees of freedom were considered. For the rest of the EM models of the database, a slight variation in the number of elements is expected due to changes in the design parameters. 
Table 2. Properties of materials

\begin{tabular}{lcc}
\hline Property & Steel & Rubber \\
\hline Elastic modulus $[\mathrm{Pa}]$ & $200 \times 10^{9}$ & $1.5 \times 10^{6}$ \\
\hline Poisson's ratio & 0.29 & 0.45 \\
\hline Density $\left[\mathrm{kg} / \mathrm{m}^{3}\right]$ & 7850 & 1100 \\
\hline
\end{tabular}

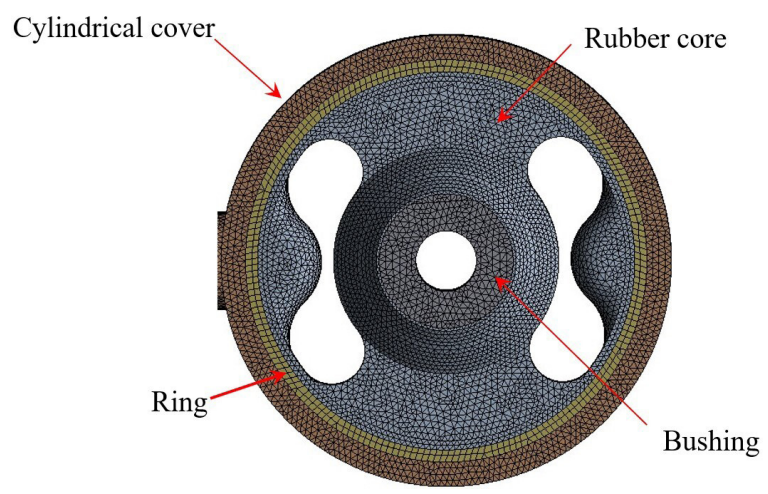

Fig. 6. Meshed domain for the EM geometrical model

The total engine weight is distributed and applied as a force among the three EM that support the engine in the engine bay. So, a force of $457.83 \mathrm{~N}$ was calculated using the engine mass of $140 \mathrm{~kg}$, and then it was applied at the internal face of the EM bushing. Furthermore, the harmonic response requires applying a dynamic force, which was considered as three times the force applied to EM bushing resulting in a final force of $1373.5 \mathrm{~N}$. Two displacement restrictions were applied, one at the inner face of the bushing of the part bolted to chassis, the second one at the external surface of the cylindrical cover. No sliding or separation was considered for the contact between the EM parts.

The harmonic response analysis was performed in a range of $5 \mathrm{~Hz}$ to $30 \mathrm{~Hz}$ with frequency steps of 1 $\mathrm{Hz}$. At each frequency step, resultant deformation and stress responses were calculated.

\subsection{Artificial Neural Networks}

An ANN works as an interpolator in the classification process and static or time-series predictions. A type of feed-forward ANN, widely used in prediction is the multilayer perceptron (MLP), which has an architecture of layers arranged in input, hidden, and output layers. Each layer is composed of a defined number of neurons which, in the case of input and output layers, corresponds to the number of inputs and predicted outputs, respectively. In the case of the hidden layer, the number of neurons is defined by trial and error, until it reaches an acceptable reliability prediction. Each layer is linked to the next one through weighted connections. To establish nonlinear relationships between inputs and outputs, transfer functions must be added to ANN. A good performance in prediction could be found using the hyperbolic tangent (HT) function between input and hidden layer, whereas between the hidden and output layers, a linear function could be used [20] to [23]. The predicted output is calculated through:

$$
y=l\left(\sum_{j=1}^{n}\left(w_{j}^{2} \cdot H T_{n}+b^{2}\right)\right),
$$

where $l$ is the linear function, $w$ is the weight connection, $H T$ is the hyperbolic tangent transfer function, $b$ is the bias, $n$ is the total number of hidden neurons. The $H T$ is:

$$
H T_{n}=\frac{2}{1+e^{-2 x_{n}}}-1,
$$

where $x$ is defined as:

$$
x_{n}=\sum_{i=1}^{m} w_{i}^{1} \cdot i n_{i}+b_{j}^{1},
$$

where $m$ is the number of neurons in the input layer. The superscripts 1 and 2 indicate inputs to the hidden layers and outputs from the hidden layers, respectively. Weights and biases coefficients are obtained from the ANN training process and inserted in the above equations to calculate the desired output. The adequate selection of these coefficients is made by a training algorithm. To train, validate, and test the ANN, the database of section 1.4 was used.

In this work, three ANN models were implemented to calculate stress and displacements response at a specified frequency range and, due to the complex shape, the mass of the rubber core of the EM. The architecture of all three models consists of three layers with eight and one neurons at input and output layers, respectively. The model to predict the stress response area has six neurons in the hidden layer, whereas the model for displacements has three neurons, and just two neurons in the hidden layer for the mass prediction. The database to train the ANN consists of 80 design combinations based on a central composite design of experiments and an additional baseline design. This design of experiments was implemented to avoid duplicated information which difficult the ANN learning. The database was constructed by FEM simulations of the harmonic response of EM subjected to excitation frequencies. All design combinations of the database show the same trend increasing displacement and stress responses as frequency increases. The net was trained using the Levenberg-Marquardt algorithm, which has shown 
a good prediction performance compared with other training algorithms [24]. The transfer functions used in these models were HT, between input and hidden layer, and linear, between hidden and output layer. Due to the use of HT, the input data were normalized between 0.1 and 0.9 using the next expression [25]:

$$
n_{i}=0.8\left(\frac{I n_{i}-I n_{\min }}{I n_{\max }-I n_{\min }}\right)+0.1 .
$$

Here $n_{i}$ is the normalized variable, $I n_{i}$ is the notnormalized variable, and $I n_{\min }$ and $I n_{\max }$ are the lower and upper range of the design variable.

The performance of the ANN models was evaluated using the estimation of both root mean square error, RMSE, and the correlation coefficient, $R^{2}$, calculated as follows:

$$
\begin{gathered}
R M S E=\sqrt{\frac{\sum_{t=1}^{T}\left(y_{A N N}-y_{F E M}\right)^{2}}{T},} \\
R^{2}=1-\frac{\sum_{t=1}^{T}\left(y_{A N N}-y_{F E M}\right)^{2}}{\sum_{t=1}^{T}\left(y_{F E M}-y_{\text {ave }}\right)},
\end{gathered}
$$

where $y_{A N N}$ is the output predicted by ANN, $y_{F E M}$ is the output predicted by FEM, $y_{\text {ave }}$ is the average of actual values. From the database, $80 \%$ of data were randomly selected to train the net, whereas the rest of the data were used in the validation process. To test the ANN models, eight additional simulations (10\% of the database) were computed to evaluate ANN predictions against FEM calculations.

\subsection{Optimization}

The proposed optimization is based on the GODLIKE algorithm developed by Oldenhuis and Vandekerckhove [26]. GODLIKE uses four single meta-heuristic algorithms: differential evolution (DE), genetic algorithm (GA), particle swarm optimization (PSO) and adaptive simulated annealing (ASA). In GODLIKE, each algorithm performs the first approach from an initial population. Before starting a second approach, a defined number of members of the firstly optimized population is randomly selected from one algorithm (e.g., DE) and inserted in the population of the remaining algorithms (e.g., GA, PSO, and ASA). The second approach starts until all the algorithms have shared members among them. This process is repeated until a stop condition is met. In this way, the possible poor performance of each algorithm is improved due to the integration of population members from other algorithm approaches.
This link between algorithms is intended to find the global optimum due to the use of populations with the fittest individuals. The use of this methodology requires many objective function evaluations, which increase the computational cost; however, it is offset by the robustness of the algorithm [27].

\subsection{Objective Function}

A multi-objective optimization works by minimizing or maximizing several objective functions that satisfy a defined set of constraints [28]. The problem can be mathematically written as:

$$
\text { minimize }\left\{f_{1}(\vec{X}), f_{2}(\vec{X}), \ldots, f_{N}(\vec{X})\right\},
$$

subject to; $g_{j}(\vec{X}) \leq 0, h_{k}(\vec{X})=0$, and $x_{l b} \leq x_{M} \leq x_{u b}$. Here $f$ is the objective function, $\vec{X}$ is a vector that contains the design variables $x, g$ and $h$ are the inequality and equality constraint functions, respectively. The subscript $N$ is the total of objective functions; $j, k$ and $M$ are the amounts of inequalities constraints, equalities constraints, and design variables respectively, whereas $l_{b}$ and $u_{b}$ are the lower and upper bounds of the corresponding design variable. When a multi-objective optimization is performed, more than one solution is obtained; these feasible solutions lie on the Pareto-optimal front [29].

In this work, the total mass reduction of the EM was calculated by adding the mass of all its parts: rubber core, ring, metal cover, and the bushing. Due to the complex shape of the rubber core, its mass was computed using an ANN model, whereas the mass of the rest of the components was evaluated as follows:

$$
\begin{gathered}
m_{r u}=m_{r u, A N N}, \\
m_{r i}=\pi 62.8\left(V_{1}^{2}-V_{2}^{2}\right), \\
m_{m c}=\pi 62.8\left(V_{4}^{2}-V_{1}^{2}\right), \\
m_{b u}=\pi 90.275\left(V_{3}^{2}-0.010^{2}\right) .
\end{gathered}
$$

Here $m_{r u}$ is the mass of the rubber part, and the subscript $A N N$ indicates the rubber mass predicted by means of the neural net. Deformations and stresses vary as functions of the mechanical vibrations and their excitation frequencies. Since the engine works at different frequencies, a method was implemented to evaluate both displacement and stress as response areas considering a frequency step of $S=1 \mathrm{~Hz}$. The method aims to calculate every response area as an area of a trapezoidal shape formed between two 
consecutive frequency steps. For this purpose, the coordinates of four points must be in a plane where frequencies versus response (displacement or stresses) are plotted at the $x$-axis and $y$-axis, respectively. The coordinates of the four points are defined by their position at $x$-axis and $y$-axis, respectively. For example, in Fig. 7, the displacement response area A is formed by points $1,2,3$, and 4 . The coordinates of point 1 and 2 at the $x$-axis are the frequency $\omega$ at step 1 and frequency $\omega+1$ respectively, whereas at the $y$-axis its coordinate is 0 . The coordinates of points 3 and 4 at the $x$-axis are the same as in points 1 and 2; at the $y$-axis, the coordinates correspond to response magnitude at $\omega$ and $\omega+1$, respectively. Then, the four points are connected, closing the profile forming a trapezoidal area, which evaluates the response through two consecutive frequencies.

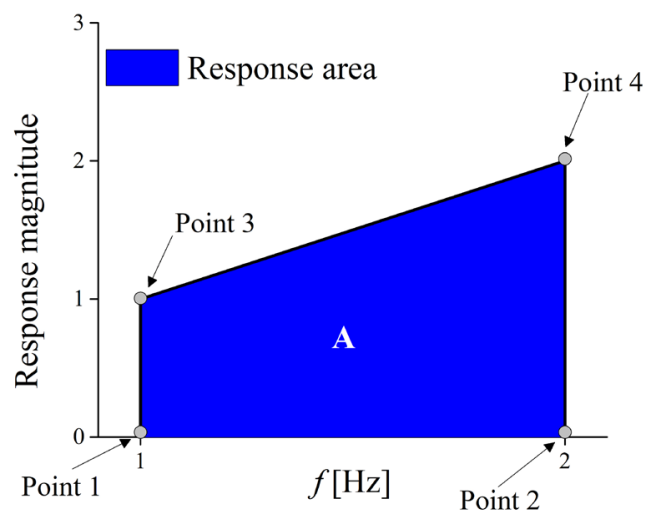

Fig. 7. Definition of coordinates at the response area

The response areas for displacement and stress are calculated using Eqs. (14) and (15) respectively:

$$
\begin{aligned}
& A_{d \omega}=\frac{d_{\omega+1}+d_{\omega}}{2} s, \\
& A_{\sigma \omega}=\frac{\sigma_{\omega+1}+\sigma_{\omega}}{2} s,
\end{aligned}
$$

where $A_{d \omega}$ and $A_{\sigma \omega}$ are the areas for displacement and stress responses, respectively, and $d$ and $\sigma$ are the displacement and stress responses obtained at a specific excitation frequency, $\omega$.

For the optimized design of the EM, a multiobjective optimization was raised. Three objective functions were selected with a set target for each one of them. Penalty factors were added to objective functions to define the relevance of each variable in the optimization process.

Objective functions are expressed in Eqs. (16) to (18). Eq. (16) minimizes the expression to evaluate the mass of the EM, which involves the mass of rubber part, ring, metal cover, and bushing. Eqs. (17) and (18) are the average of the response areas of displacement and stress, respectively, during the excitation frequencies range.

$$
\begin{aligned}
\min f_{1}= & \left(\frac{m_{r u}+m_{r i}+m_{m c}+m_{b u}}{m_{t a r}}\right)\left(P_{f 1}\right), \\
& \min f_{2}=\frac{A_{d \omega}^{a v g}}{A_{d \omega, \text { atar }}^{a v g}}\left(P_{f 2}\right), \\
& \min f_{3}=\frac{A_{\sigma \omega}^{a v e}}{A_{\sigma \omega, \text { atar }}^{\text {ave }}}\left(P_{f 3}\right) .
\end{aligned}
$$

Here, $A_{d \omega}^{a v g}=\sum_{i=1}^{S-1} A_{d, i} /(S-1)$ is the average area response of displacement, $A_{\sigma \omega}^{\text {avg }}=\sum_{i=1}^{S-1} A_{\sigma, i} /(S-1)$ is the area average response of stresses, whereas $S$ is the total number of excitation frequencies. The subscript tar indicates the targets which were set to reach the convergence to the optimized values. The targets were, $m_{\text {tar }}=0.45 \mathrm{~kg}, A_{\text {d,tar }}=0.0035 \mathrm{~m} \cdot \mathrm{Hz}$ and $A_{\sigma, t a r}=$ $0.5 \mathrm{MPa} \cdot \mathrm{Hz}$. The penalty factors are: $P_{f 1}, P_{f 2}$ and $P_{f 3}$ for mass, displacement response and stress response, respectively. In this work, it was assumed that passenger comfort (which is related to the displacement) is the main criterion to consider followed by the resistance of components and finally the mass of the component. Based on these assumptions, the penalty factors were set as follows: 0.15 for mass, 0.60 for displacement and 0.25 for stresses.

\subsection{Validation of FEM Model}

The FEM model was validated through the measurement of the first natural frequency of the EM. The accelerometer utilized was the same used in the measurements of engine mechanical vibrations. The device was mounted over the EM inside the engine bay. The excitation signal was provided by an impact hammer.

The experimental measurement of EM first natural frequency is shown in Fig. 8. The value of the first natural frequency was $75.015 \mathrm{~Hz}$. The first three natural frequencies from FEM computations are shown in Table 3. The comparison of both the experimental and numerical first natural frequencies shows a good agreement between measured and predicted frequencies with a difference of around 7.5 $\%$. These results guarantee the accuracy of the FEM model and its validity for the optimization of the EM. 
Table 3. Natural frequencies calculated by FEM

\begin{tabular}{lc}
\hline Natural frequency & Frequency $[\mathrm{Hz}]$ \\
\hline 1 st & 80.68 \\
\hline $2^{\text {nd }}$ & 89.68 \\
\hline 3 rd & 104.98 \\
\hline
\end{tabular}

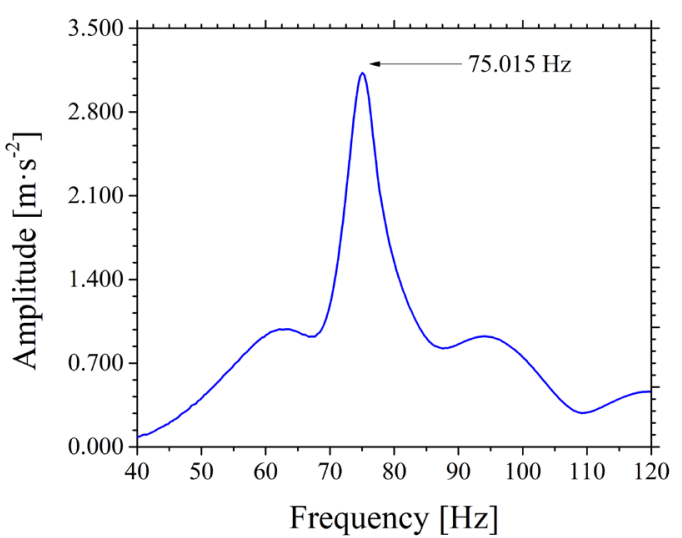

Fig. 8. Experimental measurement of the first natural frequency

\section{RESULTS AND DISCUSSION}

The results of the ANN computations are presented in Fig. 9. For $A_{\sigma \omega}^{a v g}$ training and validation, the correlation coefficient was greater than 0.98 , whereas the cases of $A_{d \omega}^{a v g}$ and $m_{r u}$ is greater than 0.99 . To test the ANN models, eight additional simulations $(10 \%$ of the database) were carried out to compare ANN predictions against FEM calculations. In the cases of displacement response and rubber mass computation, the ANN testing predictions have an error below 5\%, whereas for stress response prediction the error is slightly greater than $5 \%$. These results show the capability of the ANN models to predict reliably and ensure its use in the optimization of the EM.

The weights and biases to predict stress response, displacement response and rubber mass are presented in Tables 4 to 6 . These coefficients were obtained during ANN training and correspond to the best fit for the three predictions.

In contrast with other works, such as [16] and [18], that have used similar optimization techniques assisted by surrogated methods, this research includes experimental measurements, which guarantee that optimization was performed over operating conditions. Furthermore, considering displacement as an objective function instead of a constraint (as in [16]) allows reducing displacement along with the whole range of engine frequencies.

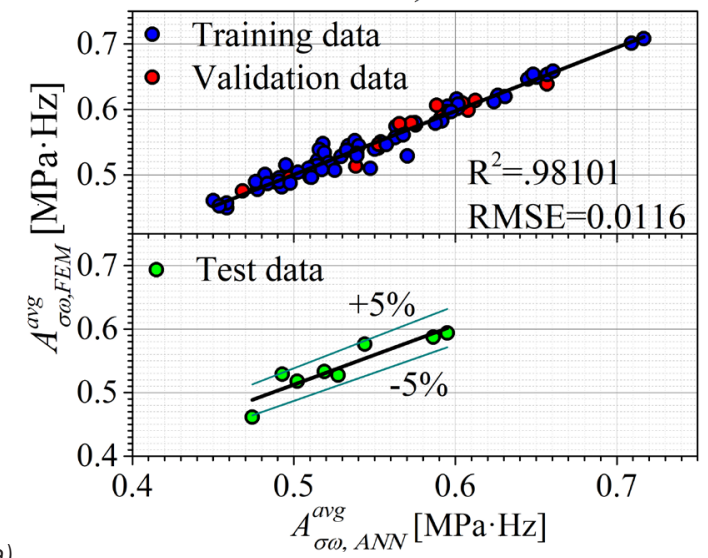

a)

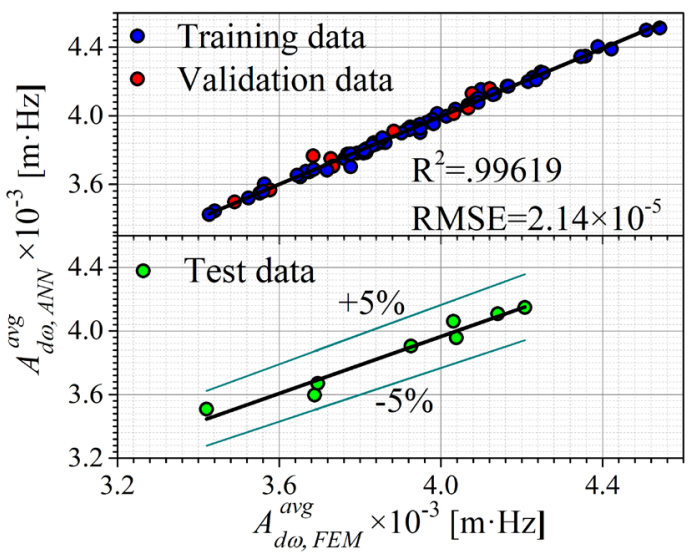

b)

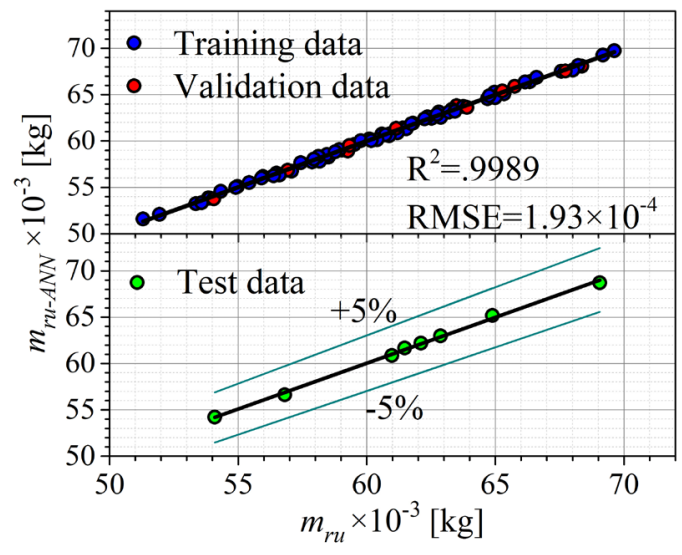

Fig. 9. Results of ANN predictions for

a) $A_{\sigma \omega}^{a v g}$, b) $A_{d \omega}^{a v g}$ and c) $m_{r u}$

In Fig. 10, the results of the optimization process are presented. Around $25 \%$ of the Pareto points improve the baseline design. Possible candidates were found with a reduced displacement; nevertheless, their mass was around $0.5 \mathrm{~kg}$ which makes them nonfeasible candidates. As the $A_{\sigma \omega}^{\text {avg }}$ and $A_{d \omega}^{\text {avg }}$ reduce their magnitude, more options can be found with a lower mass. 
Table 4. ANN coefficients for prediction of area average of stress response

\begin{tabular}{|c|c|c|c|c|c|c|c|}
\hline$w_{1,1}^{1}$ & $w_{1,2}^{1}$ & $w_{1,3}^{1}$ & $w_{1,4}^{1}$ & $w_{1,5}^{1}$ & $w_{1,6}^{1}$ & $w_{1,7}^{1}$ & $w_{1,8}^{1}$ \\
\hline-1.45854 & 0.96315 & -1.82456 & -0.49916 & -0.45800 & 5.08531 & 2.05659 & -3.16593 \\
\hline$w_{2,1}^{1}$ & $w_{2,2}^{1}$ & $w_{2,3}^{1}$ & $w_{2,4}^{1}$ & $w_{2,5}^{1}$ & $w_{2,6}^{1}$ & $w_{2,7}^{1}$ & $w_{2,8}^{1}$ \\
\hline 0.98225 & 2.23806 & -1.75895 & -1.17739 & -2.32274 & -1.14974 & 1.65331 & -2.22049 \\
\hline$w_{3,1}^{1}$ & $w_{3,2}^{1}$ & $w_{3,3}^{1}$ & $w_{3,4}^{1}$ & $w_{3,5}^{1}$ & $w_{3,6}^{1}$ & $w_{3,7}^{1}$ & $w_{3,8}^{1}$ \\
\hline 1.39160 & -5.58502 & -2.39672 & -0.12927 & -0.05249 & 1.15514 & -0.59857 & -1.20014 \\
\hline$w_{4,1}^{1}$ & $w_{4,2}^{1}$ & $w_{4,3}^{1}$ & $w_{4,4}^{1}$ & $w_{4,5}^{1}$ & $w_{4,6}^{1}$ & $w_{4,7}^{1}$ & $w_{4,8}^{1}$ \\
\hline 0.63538 & 3.30013 & -1.19526 & -0.09034 & -1.41403 & -1.35004 & -3.69895 & -2.78710 \\
\hline$w_{5,1}^{1}$ & $w_{5,2}^{1}$ & $w_{5,3}^{1}$ & $w_{5,4}^{1}$ & $w_{5,5}^{1}$ & $w_{5,6}^{1}$ & $w_{5,7}^{1}$ & $w_{5,8}^{1}$ \\
\hline-2.83462 & -1.74426 & 1.65473 & 1.03900 & 3.66780 & -0.30788 & -0.69853 & 2.18040 \\
\hline$w_{6,1}^{1}$ & $w_{6,2}^{1}$ & $w_{6,3}^{1}$ & $w_{6,4}^{1}$ & $w_{6,5}^{1}$ & $w_{6,6}^{1}$ & $w_{6,7}^{1}$ & $w_{6,8}^{1}$ \\
\hline 0.17086 & -1.81009 & 0.84667 & 0.12948 & -2.15127 & 4.82269 & 0.90327 & -0.18045 \\
\hline$w_{1,1}^{2}$ & $w_{1,2}^{2}$ & $w_{1,3}^{2}$ & $w_{1,4}^{2}$ & $w_{1,5}^{2}$ & $w_{1,6}^{2}$ & & \\
\hline 0.03196 & -0.07535 & 0.16155 & 0.06778 & -0.53198 & 0.12223 & & \\
\hline$b_{1,1}^{1}$ & $b_{2,1}^{1}$ & $b_{3,1}^{1}$ & $b_{4,1}^{1}$ & $b_{5,1}^{1}$ & $b_{6,1}^{1}$ & & \\
\hline 1.15072 & 2.51253 & 0.89235 & 2.00106 & -5.08572 & -3.36807 & & \\
\hline \multicolumn{8}{|l|}{$b_{1,1}^{2}$} \\
\hline
\end{tabular}

Table 5. ANN coefficients for prediction of area average of displacement response

\begin{tabular}{|c|c|c|c|c|c|c|c|}
\hline$w_{1,1}^{1}$ & $w_{1,2}^{1}$ & $w_{1,3}^{1}$ & $w_{1,4}^{1}$ & $w_{1,5}^{1}$ & $w_{1,6}^{1}$ & $w_{1,7}^{1}$ & $w_{1,8}^{1}$ \\
\hline-0.11114 & -0.06945 & 6.66939 & -0.11978 & 0.05504 & 0.22898 & 0.31355 & -0.27377 \\
\hline$w_{2,1}^{1}$ & $w_{2,2}^{1}$ & $w_{2,3}^{1}$ & $w_{2,4}^{1}$ & $w_{2,5}^{1}$ & $w_{2,6}^{1}$ & $w_{2,7}^{1}$ & $w_{2,8}^{1}$ \\
\hline 0.01930 & -0.08350 & -0.02500 & 0.04518 & 0.03851 & -0.53731 & 0.52415 & 0.18607 \\
\hline$w_{3,1}^{1}$ & $w_{3,2}^{1}$ & $w_{3,3}^{1}$ & $w_{3,4}^{1}$ & $w_{3,5}^{1}$ & $w_{3,6}^{1}$ & $w_{3,7}^{1}$ & $w_{3,8}^{1}$ \\
\hline 0.01093 & 3.65897 & -1.03528 & 0.72872 & 0.91272 & -0.74952 & 0.14733 & -0.06379 \\
\hline$w_{1,1}^{2}$ & $w_{1,2}^{2}$ & $w_{1,3}^{2}$ & & & & & \\
\hline-0.00018 & -0.00162 & 0.00010 & & & & & \\
\hline$b_{1,1}^{1}$ & $b_{2,1}^{1}$ & $b_{3,1}^{1}$ & & & & & \\
\hline-2.58080 & 0.32367 & -2.47434 & & & & & \\
\hline \multicolumn{8}{|l|}{$b_{1,1}^{2}$} \\
\hline 0.00464 & & & & & & & \\
\hline
\end{tabular}

Table 6. ANN coefficients for rubber mass prediction

\begin{tabular}{cccccccc}
\hline$w_{1,1}^{1}$ & $w_{1,2}^{1}$ & $w_{1,3}^{1}$ & $w_{1,4}^{1}$ & $w_{1,5}^{1}$ & $w_{1,6}^{1}$ & $w_{1,7}^{1}$ & $w_{1,8}^{1}$ \\
0.00051 & -0.47488 & 0.21294 & 0.00173 & 0.03700 & 0.31568 & -0.11993 & -0.12761 \\
\hline$w_{2,1}^{1}$ & $w_{2,2}^{1}$ & $w_{2,3}^{1}$ & $w_{2,4}^{1}$ & $w_{2,5}^{1}$ & $w_{2,6}^{1}$ & $w_{2,7}^{1}$ & $w_{2,8}^{1}$ \\
1.11450 & 2.60450 & -0.06103 & 1.59204 & 0.57664 & -0.56463 & -0.80459 & -1.36578 \\
\hline$w_{1,1}^{2}$ & $w_{1,2}^{2}$ & & & & & & \\
-0.03618 & 0.000068 & & & & & & \\
\hline$b_{1,1}^{1}$ & $b_{2,1}^{1}$ & & & & & & \\
0.05320 & -0.93912 & & & & & &
\end{tabular}


Individual results are presented in Table 7. For the optimized case, total reductions of $21.5 \%, 12.46$ $\%$, and $15.3 \%$ were obtained for $A_{\sigma \omega}^{\text {avg }}, A_{d \omega}^{a v g}$ and mass, respectively, regarding the baseline design. These reductions are attributable to the application of targets and penalty factors. $A_{d \omega}^{\text {avg }}$ is considered the most important parameter, and it was set with a penalty factor of $60 \%$; however, the magnitude of its reduction was lower than others resulting in the hardest parameter to optimize.

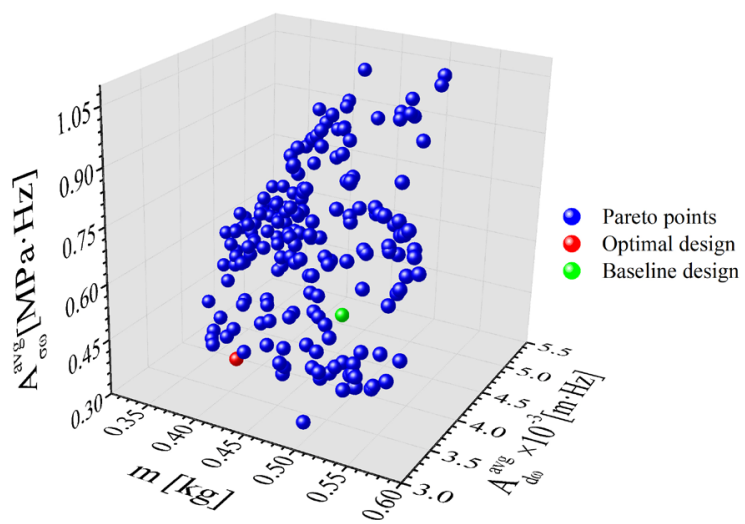

Fig. 10. Pareto points, optimal design found and baseline design

Table 7. Comparison of optimization results of $A_{\sigma \omega}^{a v g}, A_{d \omega}^{a v g}$ and mass

\begin{tabular}{ccc}
\hline & Baseline design & Optimal design \\
\hline$A_{\sigma \omega}^{\text {avg }}[\mathrm{MPa} \cdot \mathrm{Hz}]$ & 0.53042 & 0.41632 \\
\hline$A_{d \omega}^{\text {avg }}[\mathrm{m} \cdot \mathrm{Hz}]$ & 0.00385 & 0.00337 \\
\hline mass $[\mathrm{kg}]$ & 0.49148 & 0.41628 \\
\hline
\end{tabular}

Table 8. Magnitudes of the baseline and optimized design variables

\begin{tabular}{lcc}
\hline Variable & Baseline design [mm] & Optimal design [mm] \\
\hline$V_{1}$ & 66 & 67.404 \\
\hline$V_{2}$ & 62 & 61.683 \\
\hline$V_{3}$ & 22 & 22.003 \\
\hline$V_{4}$ & 74 & 72.189 \\
\hline$V_{5}$ & 2 & 1.683 \\
\hline$V_{6}$ & 59 & 57.151 \\
\hline$V_{7}$ & 38 & 39.63 \\
\hline$V_{8}$ & 37 & 38.736 \\
\hline
\end{tabular}

In Table 8, the optimized dimensions of EM are presented. The loss of mass is due to the reduction of the thickness of the wall of the cylindrical cover and the internal diameter of ring. The increment of $V_{1}$ combined with the reduction of $V_{2}$ causes the increment of internal ring wall thickness. Due to the increment of the ring wall thickness, the movement of the rubber core is restricted, thereby reducing the displacements. The length of $V_{7}$ increases the strengthening of the rubber core thus achieving a reduction of the stress response.

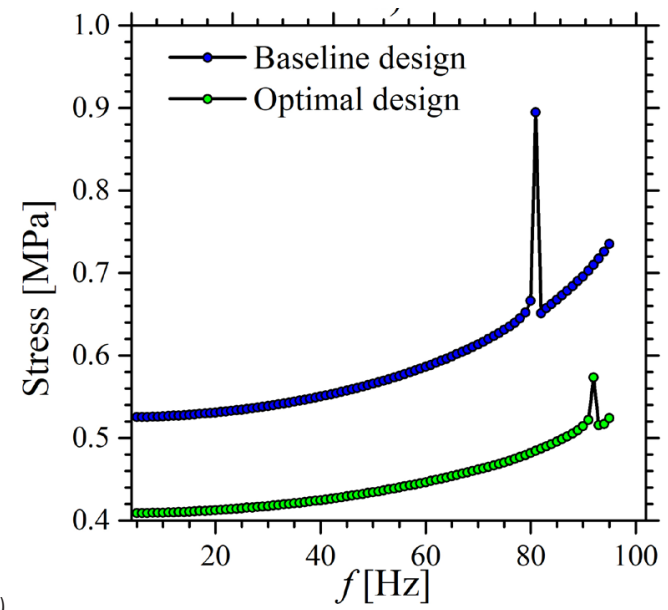

a)

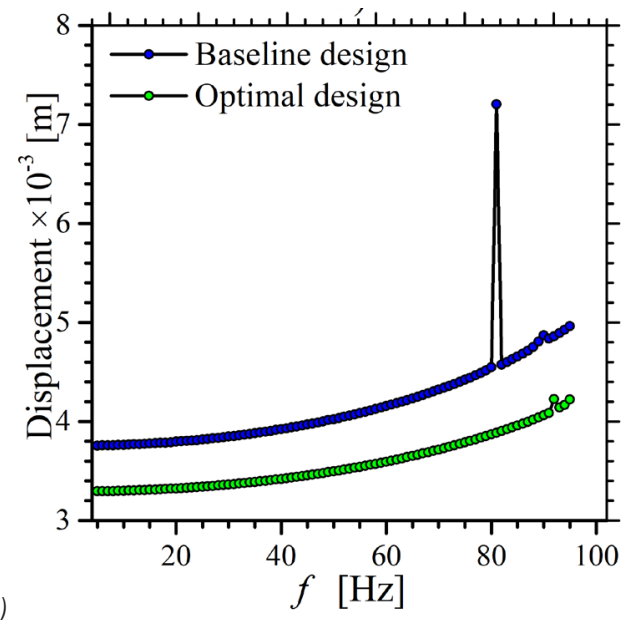

Fig. 11. Comparison of responses through the frequency range, a) stress, b) displacement

With the optimized design variables, a new geometrical model, which corresponds to optimized EM, was generated. For such optimized geometry, new computations of the frequency response were performed and compared against the baseline design. In Fig. 11, stress and displacements are plotted against the typical $(5 \mathrm{~Hz}$ to $30 \mathrm{~Hz})$ and extended $(30 \mathrm{~Hz}$ to $100 \mathrm{~Hz}$ ) excitation frequency of the internal combustion engine. The improvement of the optimized EM is outstanding due to the difference in stress and displacement responses between baseline and the optimal design. For the case of the baseline design, the difference between the computed amplitude of displacement response for the initial (5 
$\mathrm{Hz}$ ) and the last $(30 \mathrm{~Hz})$ frequencies was $0.0896 \times 10^{-3}$ $\mathrm{m}$. While for the case of the optimized design, such a difference was reduced to $0.06839 \times 10^{-3} \mathrm{~m}$. Also, the stress response amplitude had a reduction of $35.9 \%$ between the baseline design case and the optimized case. These results are significant when a vehicle is accelerating between this frequency range because, in the case of displacements, a smaller movement will be transmitted to vehicle chassis affecting passenger comfort, whereas, in the case of stresses, its amplitude is minor, reducing the effects of fatigue in the EM. Extending the frequency excitation range, the difference between displacement and stress responses (Fig. 11) for the case of baseline and optimized EM is increased. Also, Fig. 11 shows that the peak which corresponds to the first natural frequency is decreased for the case of the optimized model. This contributes to reducing the amplitude of vibrations of the vehicle chassis in case of resonance at that frequency.

The distribution of stresses at $21 \mathrm{~Hz}, 2500 \mathrm{rpm}$, is presented in Fig. 12. Stresses are concentrated in the region near the rubber neck. Fig. 12a shows how the magnitude of stresses is greater than the optimized EM for which a reduction is observed at this zone. Around the external surface of the rubber core, the stresses are lower than in the centre region. A stress line extends out from the front of the neck to the back. Thereby, if a failure occurs, it is expected to be in this zone. These findings are consistent with those presented in Fig. 1, which shows the cracks of a failed EM.

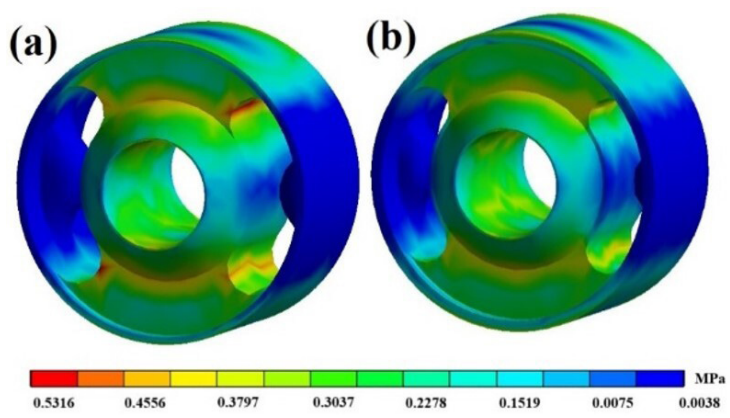

Fig. 12. Comparison of stress distributions at $21 \mathrm{~Hz}(2500 \mathrm{rpm})$, a) baseline design, b) optimized design

A comparison of displacements is presented in Fig. 13 at $21 \mathrm{~Hz}$ equivalent to $2500 \mathrm{rpm}$. Maximum displacements are observed in the surface around the bushing and in the cone. In the optimized EM, the displacements are reduced mainly at the sides of the cone and, in a smaller proportion, at the top and bottom.

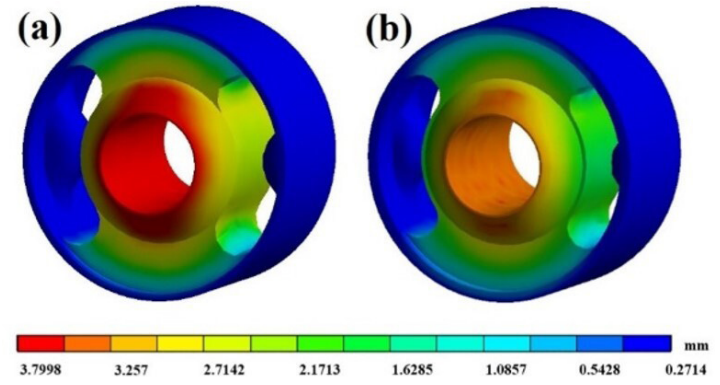

Fig. 13. Comparison of displacement at $21 \mathrm{~Hz}$ at $2500 \mathrm{rpm}$, a) baseline design, b) optimal design

\section{CONCLUSIONS}

An EM with a rubber core was optimized using a methodology that includes optimization algorithms, ANN and FEM. The optimization is focused on the reduction of EM mass, and both displacements and stresses responses under the excitation of mechanical vibration due to the engine operation. The EM mass has a reduction of $15.3 \%$, the and were reduced by $21.5 \%$ and $12.46 \%$, respectively. The target values added in the objective functions were reached, leading the optimization process toward better solutions than a baseline design. A lower magnitude of displacement and stress responses was reached, increasing the ring wall thickness and the distance between lobes in the rubber core, respectively. The reduction of the and means lowering the amplitude of stress and displacements levels in the whole frequencies range, which, in turn, reduces the displacement transmitted from engine to chassis and increases the lifetime of EM. In contrast, the mass reduction decreases production costs. The results from training, testing, and validation of the ANN assure a high confidence level in the predictions. The good definition of ANN models contributes to simplifying the optimization process in this research. The integration of the optimization methodology here presented could be applied for many optimization problems in engineering. The frequency response computation with the extended frequency range shows that the proposed optimization method of averaging both displacement and stress responses is effective because the trend of displacement and stresses response improvement remains continual throughout the extended range.

\section{REFERENCES}

[1] Shangguan, W.-B., Liu, X.-A., Lv, Z.-P., Rakheja, S. (2016). Design method of automotive powertrain mounting system 
based on vibration and noise limitations of vehicle level. Mechanical Systems and Signal Processing, vol. 76-77, p. 677-695, D0l:10.1016/J.ymssp.2016.01.009.

[2] Azammi, A.M.N., Sapuan, S.M., Ishak, M.R., Sultan, M.T.H. (2018). Conceptual design of automobile engine rubber mounting composite using triz-morphological chart-analytic network process technique. Defence Technology, vol. 14, no. 4, p. 268-277, D0l:10.1016/j.dt.2018.05.009.

[3] Liu, X.-A., Shangguan, W.-B., Lv, Z.-P., Ahmed, W., Zhu, W. (2017). A study on optimization method of a powertrain mounting system with a three-cylinder engine. Proceedings of the Institution of Mechanical Engineers, Part C: Journal of Mechanical Engineering Science, vol. 231, no. 12, p. 22352252, DOI:10.1177/0954406216631004.

[4] Idrees, M., Rajendra Prasad, V.B.S. (2018). Response of rubber based engine mounts with sbr as the core rubber. IOP Conference Series: Materials Science and Engineering, vol. 455, p. 012108, D0I:10.1088/1757-899x/455/1/012108.

[5] Ramachandran, T., Padmanaban, K.P. (2013). Minimization of IC engine rubber mount displacement using genetic algorithm. The International Journal of Advanced Manufacturing Technology, vol. 67, p. 887-898, D0l:10.1007/s00170-0124533-1.

[6] Tang, N., Soltani, P., Pinna, C., Wagg, D., Whear, R. (2018). Ageing of a polymeric engine mount investigated using digital image correlation. Polymer Testing, vol. 71, p. 137-144, DOI:10.1016/j.polymertesting.2018.08.036.

[7] Kim, W.D., Lee, H.J., Kim, J.Y., Koh, S.-K. (2004). Fatigue life estimation of an engine rubber mount. International Journal of Fatigue, vol. 26, no. 5, p. 553-560, D0l:10.1016/j. ijfatigue.2003.08.025.

[8] Pérez-Carabaza, S., Scherer, J., Rinner, B., López-Orozco, J.A., Besada-Portas, E. (2019). UAV trajectory optimization for minimum time search with communication constraints and collision avoidance. Engineering Applications of Artificial Intelligence, vol. 85, p. 357-371, D0I:10.1016/j. engappai.2019.06.002.

[9] Daróczy, L., Janiga, G., Thévenin, D. (2018). Computational fluid dynamics based shape optimization of airfoil geometry for an H-rotor using a genetic algorithm. Engineering Optimization, vol. 50, no. 9, p. 1483-1499, Dol:10.1080/0305 215X.2017.1409350.

[10] Cheng, J., Wang, L., Xiong, Y. (2018). An improved cuckoo search algorithm and its application in vibration fault diagnosis for a hydroelectric generating unit. Engineering Optimization, vol. 50, no. 9, p. 1593-1608, D0l:10.1080/0305 215X.2017.1401067.

[11] Joly, M.M., Verstraete, T., Paniagua, G. (2013). Differential evolution based soft optimization to attenuate vane-rotor shock interaction in high-pressure turbines. Applied Soft Computing, vol. 13, no. 4, p. 1882-1891, Dol:10.1016/j. asoc.2012.12.005.

[12] Rai, P., Barman, A.G. (2018). Design optimization of spur gear using SA and RCGA. Journal of the Brazilian Society of Mechanical Sciences and Engineering, vol. 40, art. ID 257, DOl:10.1007/s40430-018-1180-y.

[13] Yu, Q., Cai, M., Shi, Y., Fan, Z. (2014). Optimization of the energy efficiency of a piston compressed air engine. Strojniški vestnik - Journal of Mechanical Engineering, vol. 60, no. 6, p. 395-406, D0I:10.5545/sv-jme.2013.1383.

[14] Sbayti, M., Bahloul, R., BelHadjSalah, H., Zemzemi, F. (2018). Optimization techniques applied to single point incremental forming process for biomedical application. The International Journal of Advanced Manufacturing Technology, vol. 95, p. 1789-1804, D0l:10.1007/s00170-017-1305-y.

[15] Ahn, Y.-K., Kim, Y.-C., Yang, B.-S., Ahmadian, M., Ahn, K.-K., Morishita, S. (2005). Optimal design of an engine mount using an enhanced genetic algorithm with simplex method. Vehicle System Dynamics, vol. 43, no. 1, p. 57-81, D0l:10.1080/0042 3110412331290400.

[16] Lee, J.S., Kim, S.C. (2007). Optimal design of engine mount rubber considering stiffness and fatigue strength. Proceedings of the Institution of Mechanical Engineers, Part D: Journal of Automobile Engineering, vol. 221, no. 7, p. 823-835, DOI:10.1243/09544070JAUT0433.

[17] Zhao, Q., Chen, X., Wang, L., Zhu, J., Ma, Z.-D., Lin, Y. (2015). Simulation and experimental validation of powertrain mounting bracket design obtained from multi-objective topology optimization. Advances in Mechanical Engineering, vol. 7, no. 6, p. 1-10, Dol:10.1177/1687814015591317.

[18] Alvarado-Iniesta, A., Guillen-Anaya, L.G., Rodríguez-Picón, L.A., Ñeco-Caberta, R. (2020). Multi-objective optimization of an engine mount design by means of memetic genetic programming and a local exploration approach. Journal of Intelligent Manufacturing, vol. 31, p. 19-32, D0l:10.1007/ s10845-018-1432-9.

[19] Thorby, D. (2008). Structural Dynamics and Vibration in Practice, Thorby, D. (ed.), Butterworth-Heinemann, Oxford, p. 77-97.

[20] Bartecki, K. (2013). A general transfer function representation for a class of hyperbolic distributed parameter systems. International Journal of Applied Mathematics and Computer Science, vol. 23, no. 2, p. 291-307, D0l:10.2478/amcs-20130022.

[21] Bonakdari, H., Khozani, Z.S., Zaji, A.H., Asadpour, N. (2018). Evaluating the apparent shear stress in prismatic compound channels using the genetic algorithm based on multi-layer perceptron: A comparative study. Applied Mathematics and Computation, vol. 338, p. 400-411, D0l:10.1016/j. amc.2018.06.016.

[22] Kong, Y.S., Abdullah, S., Schramm, D., Omar, M.Z., Haris, S.M. (2019). Optimization of spring fatigue life prediction model for vehicle ride using hybrid multi-layer perceptron artificial neural networks. Mechanical Systems and Signal Processing, vol. 122, p. 597-621, D0I:10.1016/j.ymssp.2018.12.046.

[23] Durodola, J.F., Ramachandra, S., Gerguri, S., Fellows, N.A. (2018). Artificial neural network for random fatigue loading analysis including the effect of mean stress. International Journal of Fatigue, vol. 111, p. 321-332, D0l:10.1016/j. ijfatigue.2018.02.007.

[24] Hamzaoui, Y.E., Hernández, J.A., Silva-Martínez, S., Bassam, A., Álvarez, A., Lizama-Bahena, C. (2011). Optimal performance of cod removal during aqueous treatment of alazine and gesaprim commercial herbicides by direct and inverse neural network. Desalination, vol. 277, no. 1-3, p. 325337, D0l:10.1016/j.desal.2011.04.060. 
[25] Despagne, F., Massart, D.L. (1998). Neural networks in multivariate calibration. Analyst, vol. 123, no. 11, p. 157R-178R, DOI:10.1039/A805562I.

[26] Oldenhuis, R., Vandekerckhove, J. (2009). Godlike: A Robust Single-\& Multi-Objective Optimizer. from http:// www. mathworks.co.uk/matlabcentral/fileexchange/24838godlike-a-robust-single-multi-objective-optimizer, accessed on 2013-04-19.

[27] Sbayti, M., Bahloul, R., BelHadjSalah, H., Zemzemi, F. (2018). Optimization techniques applied to single point incremental forming process for biomedical application. The International
Journal of Advanced Manufacturing Technology, vol. 95, p. 1789-1804, D0l:10.1007/s00170-017-1305-y.

[28] Coello Coello, C., Lamont, G.B., Van Veldhuizen, D.A. (2007). Evolutionary Algorithms for Solving Multi-Objective Problems. Springer, New York, Dol:10.1007/978-1-4757-5184-0.

[29] Lim, J., Jang, Y.S., Chang, H.S., Park, J.C., Lee, J. (2020). Multi-objective genetic algorithm in reliability-based design optimization with sequential statistical modeling: An application to design of engine mounting. Structural and Multidisciplinary Optimization, vol. 61, p. 1253-1271, DOI:10.1007/s00158-019-02409-1. 